Check for updates

Cite this: J. Mater. Chem. C, 2021, 9, 2311

Received 28th August 2020,

Accepted 10th January 2021

DOI: $10.1039 / \mathrm{d} 0 \mathrm{tc} 04101 \mathrm{~g}$

rsc.li/materials-c

\section{Isotopic study of Raman active phonon modes in $\beta-\mathrm{Ga}_{2} \mathrm{O}_{3}^{\dagger}$}

\author{
Benjamin M. Janzen, (D) a Piero Mazzolini, (D) bc Roland Gillen, (D) d \\ Andreas Falkenstein, (D) Manfred Martin, (D) Hans Tornatzky, (D) af \\ Janina Maultzsch, (D) ${ }^{d}$ Oliver Bierwagen (D) ${ }^{b}$ and Markus R. Wagner (D) *a
}

\begin{abstract}
Holding promising applications in power electronics, the ultra-wide band gap material gallium oxide has emerged as a vital alternative to materials like GaN and SiC. The detailed study of phonon modes in $\beta-\mathrm{Ga}_{2} \mathrm{O}_{3}$ provides insights into fundamental material properties such as crystal structure and orientation and can contribute to the identification of dopants and point defects. We investigate the Raman active phonon modes of $\beta-\mathrm{Ga}_{2} \mathrm{O}_{3}$ in two different oxygen isotope compositions $\left({ }^{16} \mathrm{O},{ }^{18} \mathrm{O}\right)$ by experiment and theory: By carrying out polarized micro-Raman spectroscopy measurements on the (010) and (201) planes, we determine the frequencies of all 15 Raman active phonons for both isotopologues. The measured frequencies are compared with the results of density functional perturbation theory (DFPT) calculations. In both cases, we observe a shift of Raman frequencies towards lower energies upon substitution of ${ }^{16} \mathrm{O}$ with ${ }^{18} \mathrm{O}$. By quantifying the relative frequency shifts of the individual Raman modes, we identify the atomistic origin of all modes ( $\mathrm{Ga}-\mathrm{Ga}, \mathrm{Ga}-\mathrm{O}$ or $\mathrm{O}-\mathrm{O}$ ) and present the first experimental confirmation of the theoretically calculated energy contributions of $O$ lattice sites to Raman modes. The DFPT results enable the identification of Raman modes that are dominated by the different, inequivalent O- or Ga-atoms of the unit cell. We find that oxygen substitution on the $\mathrm{O}_{\text {III }}$ site leads to an elevated relative mode frequency shift compared to $\mathrm{O}_{1}$ and $\mathrm{O}_{\text {II }}$ sites. This study presents a blueprint for the future identification of different point defects in $\mathrm{Ga}_{2} \mathrm{O}_{3}$ by Raman spectroscopy.
\end{abstract}

\section{Introduction}

Gallium oxide $\left(\mathrm{Ga}_{2} \mathrm{O}_{3}\right)$ is a semiconductor material with an ultra-wide bandgap of about $4.8 \mathrm{eV},{ }^{1,2}$ enabling applications in power-electronics devices such as rectifiers, ${ }^{3,4}$ metal-semiconductor field-effect transistors (MESFETs), ${ }^{5,6}$ metal oxide semiconductor field-effect transistors (MOSFETs) ${ }^{3,4,7}$ or deepUV photodetectors. ${ }^{8,9}$ The material may, depending upon temperature and pressure, exist in five different polymorphs: $\alpha, \beta, \gamma, \kappa(\text { mostly referred as } \varepsilon)^{10}$ and $\delta .^{3,11-19}$ The majority of the

${ }^{a}$ Technische Universität Berlin, Institute of Solid State Physics, Hardenbergstr. 36, 10623 Berlin, Germany. E-mail: markus.wagner@physik.tu-berlin.de

${ }^{b}$ Paul-Drude-Institut für Festkörperelektronik, Leibniz-Institut im

Forschungsverbund Berlin e.V., Hausvogteiplatz 5-7, 10117 Berlin, Germany

${ }^{c}$ Department of Mathematical, Physical and Computer Sciences,

University of Parma, Viale delle Scienze 7/A, 43124 Parma, Italy

${ }^{d}$ Chair of Experimental Physics, Friedrich-Alexander Universität Erlangen-Nürnberg,

Staudtstr. 7, 91058 Erlangen, Germany

${ }^{e}$ Institute of Physical Chemistry, RWTH Aachen University, Landoltweg 2, 52074 Aachen, Germany

${ }^{f}$ Université de Toulouse, INSA-CNRS-UPS, LPCNO, 135 avenue de Rangueil, 31077 Toulouse, France

$\dagger$ Electronic supplementary information (ESI) available. See DOI: 10.1039/ dotc04101g
$\mathrm{Ga}_{2} \mathrm{O}_{3}$ related research focuses on the $\beta$-phase as it is the thermodynamically most stable phase ${ }^{11,12,18}$ and can be produced as bulk single crystals by different growth techniques such as the Czochralski method, ${ }^{20-23}$ floating-zone, ${ }^{1,24-27}$ edge-defined film fed (EFG), ${ }^{23,28}$ or Bridgman (horizontal or vertical, $\mathrm{HB}$ and $\mathrm{VB})^{29-31}$ growth methods.

\subsection{Crystal structure and electronic properties}

The crystal structure of the $\beta$-polymorph (Fig. 1) is monoclinic (space group: $\left.C_{2 \mathrm{~h}}^{3} ; C 2 / m\right)^{12,19,32}$ with lattice parameters $a=12.29 \AA$, $b=3.05 \AA, c=5.81 \AA$ and the monoclinic angle of $\beta=103.77^{\circ}$ between the crystallographic $a$ and $c$ axes. ${ }^{19}$

The commercial availability of $\beta-\mathrm{Ga}_{2} \mathrm{O}_{3}$ substrates with different surface planes enables lattice-matched strain-free homoepitaxial growth of $\beta-\mathrm{Ga}_{2} \mathrm{O}_{3}$ films with low defect densities. $^{33-40}$ Nonetheless, even in the case of homoepitaxy, different substrate orientations can result in the formation of structural defects in the deposited layers, e.g. twin domains in (100) and (201) films. ${ }^{33,38}$ In order to overcome this problem, homoepitaxial growth of $\beta-\mathrm{Ga}_{2} \mathrm{O}_{3}$ is mostly performed on (010) oriented substrates, ${ }^{34}$ although viable alternatives such as (001) homoepitaxy ${ }^{33}$ or the employment of proper offcuts in (100) oriented substrates ${ }^{41}$ were recently reported. 


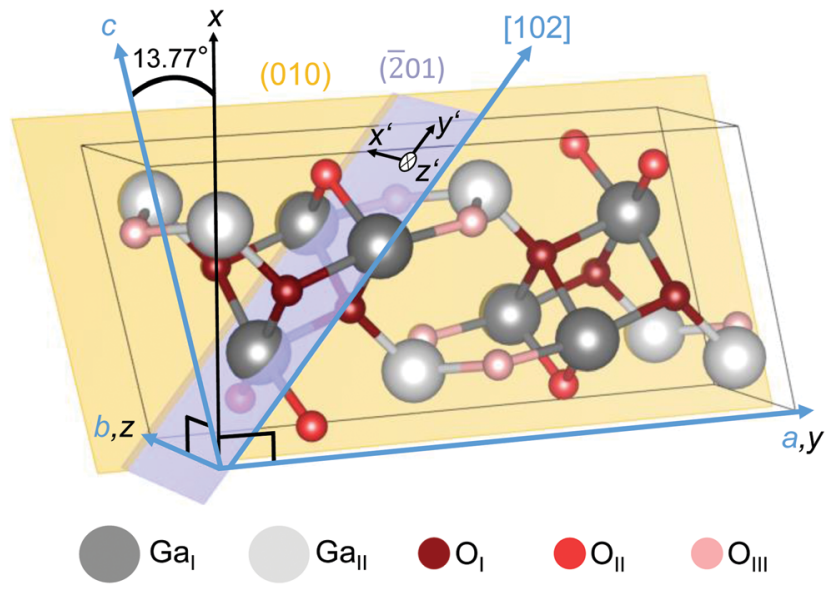

Fig. 1 Unit cell of monoclinic $\beta-\mathrm{Ga}_{2} \mathrm{O}_{3}$. Two types of $\mathrm{Ga}\left(\mathrm{G} \mathrm{a}_{1}\right.$ and $\left.\mathrm{Ga} \mathrm{a}_{\|}\right)$and three types of $O\left(O_{1}, O_{\| 1}\right.$ and $\left.O_{\| I I}\right)$ lattice sites are illustrated. By aligning the $y$ and $z$ axes of a Cartesian coordinate system (black) along the crystallographic $a$ and $b$ axes (blue), the $x$ and $c$ axes confine an angle of $13.77^{\circ} .^{19}$ The (010) plane (yellow) is spanned by the $a$ and $c$ axes. Cartesian coordinates $x^{\prime} \| b$ and $y^{\prime} \|[102]$ parameterize the (201) plane (violet), with $z^{\prime}$ denoting the surface normal. The crystallographic plot was created using VESTA. ${ }^{57}$

Previous works have demonstrated the possibility of extrinsic $\mathrm{n}$-doping by substituting $\mathrm{Ga}$ atoms with electron donors like $\mathrm{Si}^{42}$ $\mathrm{Sn}^{43} \mathrm{Ge}^{44}$ and $\mathrm{Nb}^{45}$ To date, the reported charge carrier densities in $\beta-\mathrm{Ga}_{2} \mathrm{O}_{3}$ bulk crystals ${ }^{46,47}$ and thin films ${ }^{44,45,48}$ have rarely exceeded high $10^{19} \mathrm{~cm}^{-3}$. Free carrier concentrations are commonly limited by point defects and structural defects. Theoretical works ${ }^{49-56}$ have addressed the effects of $\mathrm{Ga}$ and $\mathrm{O}$ point defects in gallium oxide on the electrical and optical properties. Using hybrid functional calculations, it was revealed that oxygen vacancies $\left(\mathrm{V}_{\mathrm{O}}\right)$ are deep donors with ionization energies above $1 \mathrm{eV}$ and hence do not contribute to the observed n-type conductivity in unintentionally doped $\beta-\mathrm{Ga}_{2} \mathrm{O}_{3} \cdot{ }^{49}$ Succeeding studies found that all other native defects, except for gallium interstitials $\left(\mathrm{Ga}_{i}\right)$, are deep, too. ${ }^{53-55}$ While $\mathrm{Ga}_{i}$ may act as shallow donors, their high mobility, coupled with large formation energies under n-type conditions inhibit their contribution to the electrical conductivity. ${ }^{56}$

Whereas intrinsic defects should not be the main source of electrical conductivity, different impurities including $\mathrm{H}, \mathrm{Si}, \mathrm{Ge}$, $\mathrm{Sn}, \mathrm{F}$ and $\mathrm{Cl}$ could act as extrinsic shallow donors. ${ }^{49,50}$ Moreover, a large concentration of point defects can also affect the mobility of free charge carriers. Nonetheless, from an experimental point of view both (i) the control over the formation of different point defects during the material synthesis and (ii) their unambiguous identification is extremely difficult to achieve, but potentially of paramount importance for fully exploiting the material potential. In order to address this challenge, we conduct a combined experimental and theoretical study of the impact of different lattice atoms on phonon modes in $\beta-\mathrm{Ga}_{2} \mathrm{O}_{3}$ isotopologues.

\subsection{Lattice dynamics}

Polarized micro-Raman spectroscopy constitutes a powerful tool that enables e.g. the study of strain in epitaxial thin films, ${ }^{58}$ the detection of dopants and point defects via local vibrational modes, ${ }^{59}$ the identification of lattice sites via angular resolved measurements, ${ }^{60}$ and the determination of the thermal conductivity via Raman thermometry in its one and two laser implementations. ${ }^{61,62}$ Symmetries and spectral positions of Raman active phonons of $\beta-\mathrm{Ga}_{2} \mathrm{O}_{3}$ in the natural ${ }^{16} \mathrm{O}$ isotopic composition have been calculated theoretically and verified experimentally in a number of publications. ${ }^{60,63-66}$ Two recent works $^{60,66}$ employed polarized micro-Raman spectroscopy to separate Raman modes of different symmetries. Infrared active phonons were investigated by IR ellipsometry. ${ }^{67}$ The influence of lattice expansion was studied by temperature dependent Raman spectroscopy of $\beta-\mathrm{Ga}_{2} \mathrm{O}_{3} \cdot{ }^{63}$ Employing the valence force field calculation, the authors indentified three categories of Raman active phonons with respect to the motions of $\mathrm{Ga}$ and $\mathrm{O}$ atoms. The transition of the $\beta$ - to the $\alpha$-phase under high pressure was investigated by carrying out Raman spectroscopy in diamond anvil cells. ${ }^{64}$ Raman tensor elements were determined in angular-resolved measurements ${ }^{60}$ using a modified Raman tensor formalism proposed in a preceding publication. ${ }^{68}$ Though the effects of isotope substitution on the frequencies of the Raman active phonon modes in $\beta-\mathrm{Ga}_{2} \mathrm{O}_{3}$ were investigated theoretically, ${ }^{69}$ no corresponding experimental study is available in the literature.

Raman vibrations may be excited by oscillations of $\mathrm{Ga}$ or $\mathrm{O}$ atoms. By calculating the displacements of the individual $\mathrm{O}$ or Ga lattice sites, density functional perturbation theory (DFPT) calculations enable the identification of the atomistic origins of vibrational modes (Ga-Ga, Ga-O or O-O). From an experimental point of view, the identification of individual lattice sites in materials with large unit cells is a challenging endeavour. A powerful tool is the usage of different isotopes and investigate their impact on the vibrational properties of a material. This approach was successfully applied in $\mathrm{TiO}_{2}$ enabling the experimental identification of Raman modes without any contribution of oxygen lattice vibrations as evidenced by the unchanged frequency of the $\mathrm{E}_{\mathrm{g}}(1)$ and $\mathrm{B}_{1 \mathrm{~g}}(1)$ modes in anatase $\mathrm{TiO}_{2}{ }^{70,71}$

Alternatively, the introduction of different isotopes of the same dopant can provide an unambiguous identification of dopant related local vibrational modes. $\mathrm{ZnO}$ is a well-studied wide band-gap oxide, whose point defects have been thoroughly investigated by vibrational spectroscopy. Apart from $\mathrm{Zn}$ and $\mathrm{O}$ vacancies, point defects in $\mathrm{ZnO}$ include $\mathrm{Zn}$ on interstitial sites $\left(\mathrm{Zn}_{\mathrm{i}}\right)$ and oxygen on interstitial sites $\left(\mathrm{O}_{\mathrm{i}}\right)^{72,73}$ Undoped $\mathrm{ZnO}$ exhibits intrinsic n-type conductivity, which is commonly attributed to one or more of the following origins: (i) intrinsic defects acting as shallow donors, ${ }^{74,75}$ (ii) shallow donors due to $\mathrm{H}$ absorbed from the air, ${ }^{76-79}$ (iii) shallow donors formed by residual trace amounts of group - III impurities such as $\mathrm{Al}, \mathrm{Ga}$ and $\mathrm{In}^{80}$ The substitution of $\mathrm{N}$ on $\mathrm{O}$ lattice sites in $\mathrm{ZnO}$ produced a pair of additional modes at $\nu \approx 274$ and $510 \mathrm{~cm}^{-1},{ }^{81-84}$ irrespective of the implanted $\mathrm{N}$ isotope. ${ }^{85,86}$ Moreover, since the doping of $\mathrm{ZnO}$ with $\mathrm{Ga}, \mathrm{Fe}, \mathrm{Sb}, \mathrm{Li}$ and $\mathrm{Al}$ yielded the same vibrational modes, ${ }^{87}$ their occurrence was tentatively attributed to a $\mathrm{Zn}_{\mathrm{i}}-\mathrm{N}_{\mathrm{i}}$ or $\mathrm{Zn}_{\mathrm{i}}-\mathrm{O}_{\mathrm{i}}$ complex. ${ }^{83}$ By investigating undoped and nitrogen-doped $\mathrm{ZnO}$ thin films in different $\mathrm{Zn}$ isotope compositions, the presence of the 
$274 \mathrm{~cm}^{-1}$ mode was eventually revealed to be related to interstitial $\mathrm{Zn}$ clusters depending on the surface polarity of $\mathrm{ZnO}{ }^{84}$ This experimental approach could represent an important milestone for the future identification of different point defects in $\mathrm{Ga}_{2} \mathrm{O}_{3}$ layers as a function of different synthesis (e.g. deposition, annealing) conditions. ${ }^{88}$ Oxygen defects such as O-vacancies and nitrogen on O-sites are deep donors and acceptors, respectively, which can strongly impact electronic behavior (traps, scattering centers, centers that get charged/discharged in space-charge devices). These defects need to be studied and we propose on the long run to use Raman spectroscopy for it.

Towards this goal, this work investigates the phonon frequencies of $\beta-\mathrm{Ga}_{2} \mathrm{O}_{3}$ in the natural ${ }^{16} \mathrm{O}$ and ${ }^{18} \mathrm{O}$ isotope compositions using confocal and cross-sectional micro-Raman spectroscopy. The acquired experimental data are complemented with the results of DFPT calculations. By quantifying the relative frequency shifts of the individual Raman modes, we discuss the origins of vibration dynamics $(\mathrm{Ga}-\mathrm{Ga}, \mathrm{Ga}-\mathrm{O}$ or $\mathrm{O}-\mathrm{O})$, resolved for different non-equivalent lattice sites, and present the first experimental confirmation of the theoretically calculated energy contribution of $\mathrm{O}$ sites to Raman modes. The results of our study shall open the possibility of analyzing O-related point defects in $\beta-\mathrm{Ga}_{2} \mathrm{O}_{3}$ by Raman spectroscopy.

\section{Experimental and theoretical methods}

A $\beta-\mathrm{Ga}_{2} \mathrm{O}_{3}$ layer was homoepitaxially deposited (deposition time $445 \mathrm{~min}$ ) on top of an unintentionally doped (010)-oriented substrate with In-mediated metal-exchange catalysis (MEXCAT) ${ }^{89-91}$ in an MBE chamber equipped with an oxygen-plasma source run at a power of $300 \mathrm{~W}$. For this deposition run, nominally $97.39 \%$ isotopically enriched ${ }^{18} \mathrm{O}_{2}$ was employed to provide an oxygen flux of 0.38 standard cubic centimeter per minute (sccm) during the layer growth. For gallium (with natural isotopic abundance) a beam equivalent pressure $(\mathrm{BEP})$ of $\mathrm{BEP}_{\mathrm{Ga}}=1.27 \times 10^{-7}$ mbar was used (equivalent to a particle flux of $\Phi_{\mathrm{Ga}}=2.2 \mathrm{~nm}^{-2} \mathrm{~s}^{-1}$ ), while the additional In-flux necessary to allow the catalytic growth of the layer at a substrate temperature of $T_{\mathrm{g}}=900{ }^{\circ} \mathrm{C}$ was set to $\Phi_{\mathrm{In}}=1 / 3 \Phi_{\mathrm{Ga}}$ $\left(\mathrm{BEP}_{\mathrm{In}}=5.2 \times 10^{-8} \mathrm{mbar}\right)$.

The ${ }^{18} \mathrm{O}$ isotope fraction, $n^{*}$, within the sample was determined by time-of-flight secondary ion mass spectrometry (ToF-SIMS) depth profiles on a ToF-SIMS IV machine (IONTOF GmbH, Münster, Germany). Measurements were performed with a $25 \mathrm{kV}$ $\mathrm{Ga}^{+}$analysis beam and a $2 \mathrm{kV} \mathrm{Cs}^{+}$sputter beam. The isotope fraction is directly accessible from the ${ }^{18} \mathrm{O}$ and ${ }^{16} \mathrm{O}$ intensities by

$$
n^{*}=\frac{I_{18} \mathrm{O}}{I_{16} \mathrm{O}+I_{18} \mathrm{O}}
$$

Crater depths were analysed by interference microscopy with a WYKO NT1100 (Veeco Instruments Inc., USA).

Raman scattering at room temperature (293 K) was excited by a $632.816 \mathrm{~nm}$ He-Ne laser on a LabRAM HR 800 spectrometer (Horiba Jobin-Yvon, France). The laser beam was focused onto the sample using a $100 \times$ Olympus objective with a numerical aperture (NA) of 0.9, with the scattered light being collected in backscattering geometry. Backreflected and elastically scattered light (Rayleigh component) was filtered using an ultra low frequency filter (ULF) unit and then spectrally-dispersed by a monochromator spectrometer with a grating of 1800 lines per $\mathrm{mm}$. The light was detected by a charge-coupled device (CCD). The spectral dispersion of the setup is $0.35 \mathrm{~cm}^{-1}$ per pixel. The spectral resolution at $650 \mathrm{~nm}\left(400 \mathrm{~cm}^{-1}\right)$ using the 1800 lines per mm grating is about $0.5 \mathrm{~cm}^{-1}$. The precision in the determination of peak positions in comparison to neighboring Raman modes after line shape fitting for non-overlapping modes is better than $0.1 \mathrm{~cm}^{-1}$. The sample was placed beneath the objective with a respective surface's normal parallel to the direction of light propagation. A $\lambda / 2$ wave plate in the excitation was set at $0^{\circ}$ or $45^{\circ}$ to polarize the incident light parallel or crossed with respect to the scattered light, which was selected using a fixed polarizer in the detection. Prior to each measurement, the Raman spectrometer was calibrated using the silicon $T_{2 \mathrm{~g}}$-peak at $520.7 \mathrm{~cm}^{-1}$.

Simulations of the lattice vibrations were performed within the frame of density functional perturbation theory (DFPT) on the level of the local density approximation (LDA) as implemented into the Quantum Espresso suite. ${ }^{92}$ The $\mathrm{Ga}(3 \mathrm{~s}, 3 \mathrm{p}, 3 \mathrm{~d})$ and the $\mathrm{O}(2 \mathrm{~s}, 2 \mathrm{p})$ states were treated as valence electrons using multi-projector optimized normconserving Vanderbildt (ONCV) pseudopotentials ${ }^{93}$ from the Pseudo Dojo repository, ${ }^{94}$ where we used a large cutoff of the planewave basis set of $180 \mathrm{Ry}$. All reciprocal space integrations were performed by a discrete $k$ point sampling of $7 \times 7 \times 7$ points in the Brillouin zone. We fully optimized the atomic positions and cell parameters of the primitive cell of $\beta-\mathrm{Ga}_{2} \mathrm{O}_{3}$ until the residual forces between atoms and the cell stress were smaller than $0.0025 \mathrm{eV} \AA^{-1}$ and $0.01 \mathrm{GPa}$, respectively. The threshold for the total energy was set to $10^{-15} \mathrm{Ry}$, which ensured tightly converged interatomic forces for the geometry optimization and of the ground state density and wavefunctions for the DFPT calculations. As a result of the intrinsic underbinding of the LDA exchangecorrelation functional, our optimized lattice constants are less than $1 \%$ underestimated compared to the experimental values (Table 1). On the other hand, it is well-known that the LDA overbinding gives rise to a typically very good agreement of the DFT phonon frequencies, ${ }^{95}$ motivating our choice for the purposes of this work.

\section{Results and discussion}

The Raman-active phonon modes of $\beta-\mathrm{Ga}_{2} \mathrm{O}_{3}$ in the ${ }^{16} \mathrm{O}$ and ${ }^{18} \mathrm{O}$ isotoplogues (substrate and film, respectively) were acquired in polarized micro-Raman measurements, the results of which are depicted in Fig. 2a-c. From ToF-SIMS experiments, the dominating presence of ${ }^{18} \mathrm{O}$ in the homoepitaxial film has been verified: the isotope fraction is $96.3 \%$ and remains constant up to a depth of $1.5 \mu \mathrm{m}$ from the surface (Fig. S2 in ESI $\dagger$ ). At the interface between film and substrate, the isotope fraction drops over an intermediate region of $300 \mathrm{~nm}$ to $0.3 \%$, which is slightly higher than the natural isotope abundance of $0.2 \%$. The 
Table 1 Lattice constants $a, b, c$ and the monoclinic angle $\beta$ obtained from DFT-LDA calculations in this work compared to experimental and theoretical data. The exchange-correlation functional applied in each theoretical work is specified

\begin{tabular}{lllll}
\hline & $a(\AA)$ & $b(\AA)$ & $c(\AA)$ & $\beta\left(^{\circ}\right)$ \\
\hline LDA $^{a}$ & 12.154 & 3.022 & 5.759 & 103.31 \\
Experiment $^{96}$ & 12.21 & 3.04 & 5.80 & 103.83 \\
AM05 $^{19}$ & 12.29 & 3.05 & 5.81 & 103.77 \\
$\mathrm{PBE}^{12}$ & 12.44 & 3.08 & 5.88 & 103.71 \\
$\mathrm{PBE}^{65}$ & 12.31 & 3.08 & 5.89 & 103.9 \\
LDA $^{97}$ & 12.23 & 3.04 & 5.80 & 103.7 \\
${ }^{a}$ This work. & & & & \\
\end{tabular}

difference might be caused by diffusion during the deposition process at elevated temperatures. The depth with $50 \%$ of the initial isotope fraction marks the interface of the film resulting in a film thickness of $1.65 \mu \mathrm{m}$ (AFM micrography of the layer reported in Fig. S1 of ESI $\dagger$ ).

The MEXCAT deposition process ${ }^{33,91,98}$ allows to widen the deposition window of $\mathrm{Ga}_{2} \mathrm{O}_{3}$ to otherwise forbidden growth regimes (e.g. the high $T_{\mathrm{g}}$ and metal-to-oxygen flux ratios used in this work), while at the same time allowing for low incorporation of the catalyzing element.

The primitive unit cell of the monoclinic structure consists of 10 atoms: $\mathrm{Ga}$ lattice sites occur in tetrahedral $\left(\mathrm{Ga}_{\mathrm{I}}\right)$ or octahedral $\left(\mathrm{Ga}_{\mathrm{II}}\right)$ coordination, with three nonequivalent oxygen sites $\left(\mathrm{O}_{\mathrm{I}}, \mathrm{O}_{\mathrm{II}}\right.$ and $\left.\mathrm{O}_{\mathrm{III}}\right)$ in between. At the $\Gamma$-point, these correspond to 27 optical phonons: ${ }^{60,63,66}$

$$
\Gamma^{\mathrm{opt}}=10 \mathrm{~A}_{\mathrm{g}}+5 \mathrm{~B}_{\mathrm{g}}+4 \mathrm{~A}_{\mathrm{u}}+8 \mathrm{~B}_{\mathrm{u}},
$$
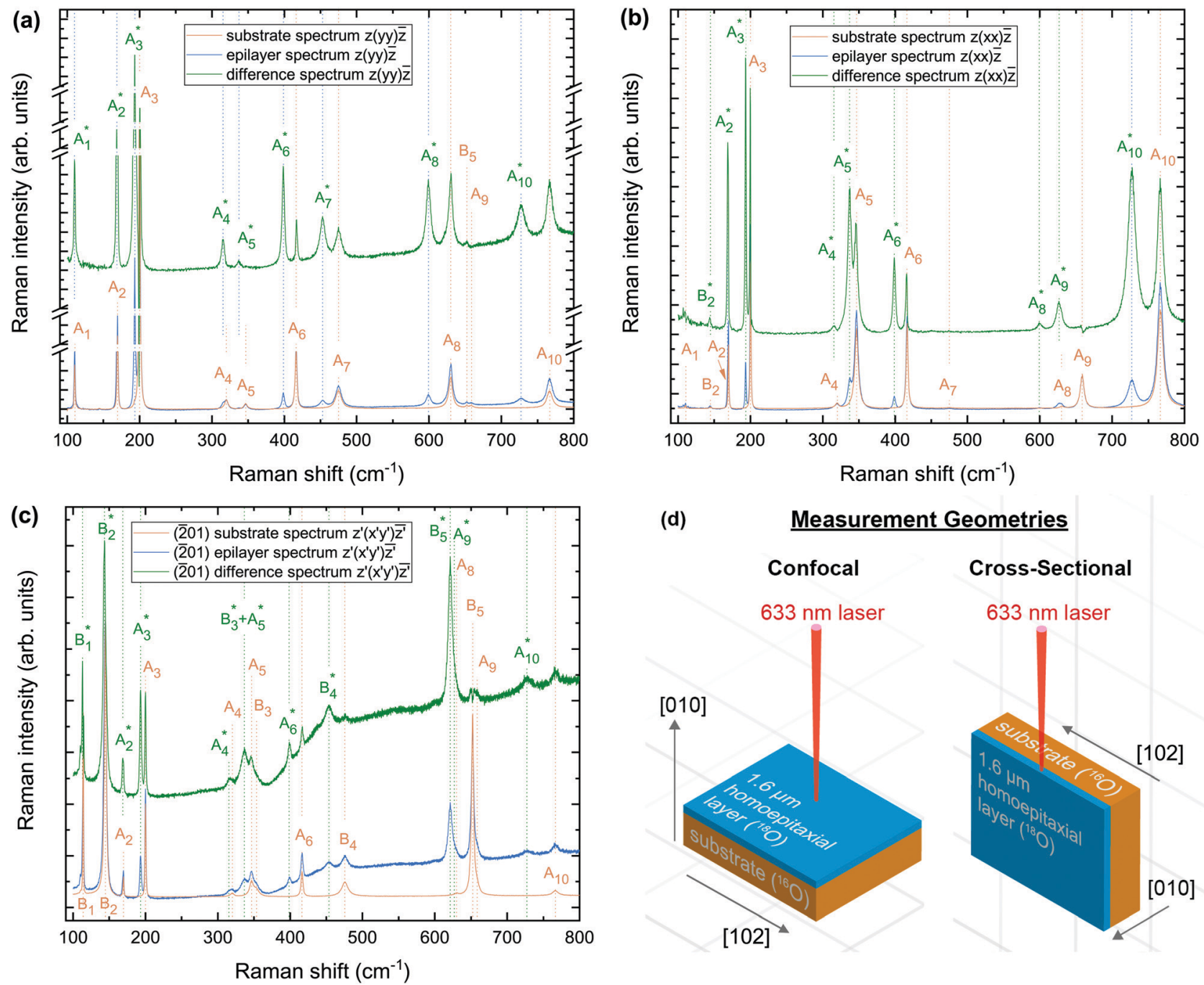

Fig. 2 Raman spectra of the homoepitaxially-grown $\mathrm{Ga}_{2} \mathrm{O}_{3}$ thin film $\left({ }^{18} \mathrm{O}\right)$ on the (010) plane of a $\beta-\mathrm{Ga}_{2} \mathrm{O}_{3}$ substrate with natural $\left({ }^{16} \mathrm{O}\right)$ isotope abundance. (a and b) Raman spectra in parallel polarization for excitation on the (010)-plane. Substrate spectra (dark yellow) were subtracted from the epilayer spectra (blue) to obtain difference spectra (green), in which ${ }^{18} \mathrm{O}$ Raman modes (denoted by *) dominate. For clarity, spectra are vertically offset and the difference spectra multiplied by a constant factor. The Cartesian coordinate system $x, y, z$ is chosen such that $z$-axis aligns with the [010] direction, while $y \|[100]$. (c) Raman spectra of the (201)plane in crossed polarization. The $z^{\prime}$-axis is parallel to the surface normal, with $x^{\prime} \|[010]$ and $y^{\prime} \|[102]$. (d) Schematic illustration of sample set-up, plane orientation, and measurement geometry. The (010) plane was accessible in confocal micro-Raman scattering (left). The [102] direction is oriented along an edge of the square-shaped wafer. Spectra of the (201)-plane were acquired by focusing the laser on the edge of the sample in cross-section geometry (right). 
15 modes are Raman-active (index g), with symmetries A and B, and 12 modes are infrared (IR) active (index $\mathrm{u}$ ).

All 15 Raman-active phonon modes of symmetries $A_{g}$ and $B_{g}$. were separated by utilization of the measurement geometries illustrated in Fig. 2d. The sample was irradiated normally in a confocal setup (left) and on the edge (right) to access the (010) or $(\overline{2} 01)$ plane, respectively. The choice of the (010) and (201) planes is advantageous as it enables the selected detection of Raman modes with $\mathrm{A}_{\mathrm{g}}$ or $\mathrm{B}_{\mathrm{g}}$ symmetry and thus facilitates the separation of modes with closely matching phonon frequencies. For the (010) plane, $\mathrm{B}_{\mathrm{g}}$-modes are forbidden as determined by Raman selection rules. For excitation normal to the (201)-plane, $A_{g}$ modes have vanishing intensity for crossed polarization of incident and scattered light when the polarization of the incident radiation aligns with the [010] direction. ${ }^{60,63}$

Fig. 2a illustrates the Raman spectra of the (010) plane in parallel polarization between incident and scattered light. Using the Porto notation, the applied scattering geometry can be written as $z(y y) \bar{z}$, where $z$ and $y$ correspond to the [010] and [100] directions, respectively ( $c f$. Fig. 1). ${ }^{60,63,66}$ In this configuration, the $\mathrm{A}_{\mathrm{g}}^{1}, \mathrm{~A}_{\mathrm{g}}^{2}, \mathrm{~A}_{\mathrm{g}}^{3}, \mathrm{~A}_{\mathrm{g}}^{7}$ and $\mathrm{A}_{\mathrm{g}}^{8}$ modes have maximum intensity. As we only investigated phonon modes accessible in Raman measurements, the index $g$ denoting a Raman-active mode is omitted in the following discussion. The Raman spectra of the substrate with ${ }^{16} \mathrm{O}$ (dark yellow) and the epilayer with ${ }^{18} \mathrm{O}$ (blue) were recorded in a confocal set-up by moving the $z$-focus into the substrate (minimum signal contribution from the film) and into the film (maximum film contribution). By subtracting the two spectra from each other, we obtained a difference spectrum (green), in which the ${ }^{18} \mathrm{O}$ Raman modes dominate. For clarity spectra are vertically offset and the difference spectrum is scaled by a constant factor. ${ }^{18} \mathrm{O}$ Raman modes are labeled with * to distinguish them from ${ }^{16} \mathrm{O}$ modes.

Subsequently, the polarization vectors of both the incident and scattered radiation were rotated $90^{\circ}$ around the [010] direction. The $x$-axis and the [001] direction confined an angle of $13.77^{\circ}$ (Fig. 1), yielding the configuration $z(x x) \bar{z}$, in which the $\mathrm{A}_{5}, \mathrm{~A}_{6}, \mathrm{~A}_{9}$ and $\mathrm{A}_{10}$ modes are maximal. The same procedures as above were applied to acquire the substrate, epilayer and difference spectra, which are displayed in Fig. $2 \mathrm{~b}$. The objective's large NA (0.9) implies a relaxation of selection rules, as light is also collected from directions other than perpendicular to the (010) plane. Hence, the most-intense $B_{2}$-mode is weakly present in Fig. $2 b$.

To access the B-modes, Raman scattering was performed in a cross-section configuration. The thickness of the film equals approximately twice the diffraction limited extent of the laser of about $800 \mathrm{~nm}$. In order to obtain the Raman spectrum of the homoepitaxial layer, we performed cross-section line scans with $200 \mathrm{~nm}$ step size. Based on these linescans, we selected two positions for long integration Raman measurements, one for which the ${ }^{18} \mathrm{O}$ related Raman modes reach maximum intensity and a second one for which only substrate modes are visible. Subtracting of the resulting spectra yielded the difference spectrum (green) in Fig. 2c.
The in-plane crystalline directions of the (010)-oriented sample were identified (i) by the presence of a rounded edge in the used substrate correspondent to the [102] crystalline direction (orthogonal to the (2̄01) planes, $c f$. Fig. 2d), (ii) by $\mathrm{X}$-ray diffraction (XRD) $2 \theta$ - and $\varphi$-scans performed on different (010) crystals, and (iii) by the recognition of (110)-facets visible as oriented lines along the [001] in-plane orientation in AFM micrographs ( $c f$. Fig. S1b, ESI $\dagger$ ). ${ }^{91,98}$ Indeed the correct probe of the (201) planes was experimentally verified via Raman spectroscopy which highlighted the mode intensity ratios expected for the $(\overline{2} 01)$ planes (Fig. 2c) ${ }^{60}$. We thus selected an edge, whose surface plane corresponds to the $(\overline{2} 01)$ plane. For normal incidence $z^{\prime}$ (cf. Fig. 1) with crossed polarization in the $z^{\prime}\left(x^{\prime} y^{\prime}\right) \bar{z}^{\prime}$ configuration $\left(x^{\prime}||[010], y^{\prime}||[102]\right)$, Raman selection rules predict vanishing intensity for the A modes and maximum intensity for the B modes. Due to the edge's imperfect preparation, A-modes were still present, yet less intense. An intensity ratio of about $3: 1$ between the most intense $B_{2}$ and $A_{3}$ modes was achieved. All five B-modes are hence available in this configuration.

The spectral positions of ${ }^{16} \mathrm{O}$ and ${ }^{18} \mathrm{O}$ Raman modes are derived from the difference spectra in Fig. 2a-c by fitting Lorentzian lineshape functions. The obtained peak positions are listed in Table 2 for both $\mathrm{O}$ isotopes in conjunction with the results of DFPT calculations. A few modes deserve particular attention, as the determination of their spectral positions and relative frequency shifts is challenging due to small frequency shifts or overlapping modes. This applies to the low-frequency $A_{1}, B_{1}, B_{2}$ and $A_{2}$ modes, for which the frequency shifts are in the order of the respective mode's linewidth.

Furthermore, in the Raman spectra of the substrate (dark yellow) shown in Fig. 2c, the $B_{3}$ resides in the right shoulder of the $\mathrm{A}_{5}$. As for the ${ }^{18} \mathrm{O}$ distribution (green), the two modes are superimposed in a joint widened peak. With the $\mathrm{A}_{5}^{*}$ position derived from Fig. 2b, we analysed this peak by setting the position of the $A_{5}^{*}$ fixed and varying the position of the $B_{3}^{*}$ until the intensity ratio $A_{3}^{*} / B_{3}^{*}$ was equal to the intensity ratio $A_{5} / B_{3}$. In the ${ }^{16} \mathrm{O}$ substrate spectrum the $\mathrm{B}_{5}$ is closely neighbored by the $\mathrm{A}_{9}$. The $\mathrm{B}_{5}^{*}$ envelope in the ${ }^{18} \mathrm{O}$ difference spectrum is composed of three individual modes: In addition to the $\mathrm{B}_{5}^{*}$, the $A_{9}^{*}$ as well as the $A_{8}$ mode from the substrate lie in close proximity. The intensity of the $\mathrm{A}_{8}$ can be regarded as negligible due to its suppression in the difference spectrum. In analogy to the analysis of the $\mathrm{B}_{3}^{*}$ spectral position, the $\mathrm{B}_{5}^{*}$ Raman frequency is obtained by setting the $\mathrm{A}_{9}^{*}$ position determined from the difference spectrum in Fig. $2 \mathrm{~b}$ fixed and varying the $\mathrm{B}_{5}^{*}$ position until obtaining equal intensity ratios for $A_{9}^{*} / B_{5}^{*}$ and $A_{9} / B_{5}$.

Distinguishing the $\mathrm{A}_{7}$ and $\mathrm{B}_{4}$ modes in both isotope compositions is a formidable task in the literature, as the two modes are located at nearly the same frequency. With the exception of one publication, ${ }^{66}$ previous experimental works have usually reported both modes at the same frequency or have only assigned one mode, ${ }^{60,63,64}$ whereas theoretical works have calculated a frequency difference ranging from 0.3 to $13.4 \mathrm{~cm}^{-1}$ between these two modes. ${ }^{60,63-65}$

Using a (201)-oriented $\beta-\mathrm{Ga}_{2} \mathrm{O}_{3}$ sample as reference, we obtained an intensity ratio $B_{4} / B_{2}>1 / 28$ between the $\mathrm{B}_{4}$ and $\mathrm{B}_{2}$ 
Table 2 Spectral positions of Raman peaks of the phonon modes of $\beta-\mathrm{Ga}_{2} \mathrm{O}_{3}$ in natural ${ }^{16} \mathrm{O}$ and ${ }^{18} \mathrm{O}$ isotope distribution, given in $\mathrm{cm}^{-1}$. Peak positions were determined from Fig. $2 \mathrm{a}-\mathrm{c}$ by fitting Lorentzian lineshape functions. The absolute and relative frequency shifts of each Raman mode are calculated and are given in $\mathrm{cm}^{-1}$ or \%, respectively. Relative shifts are rounded to two decimal places, all other quantities on one decimal place. Experimental findings are compared with the results of theoretical DFPT calculations

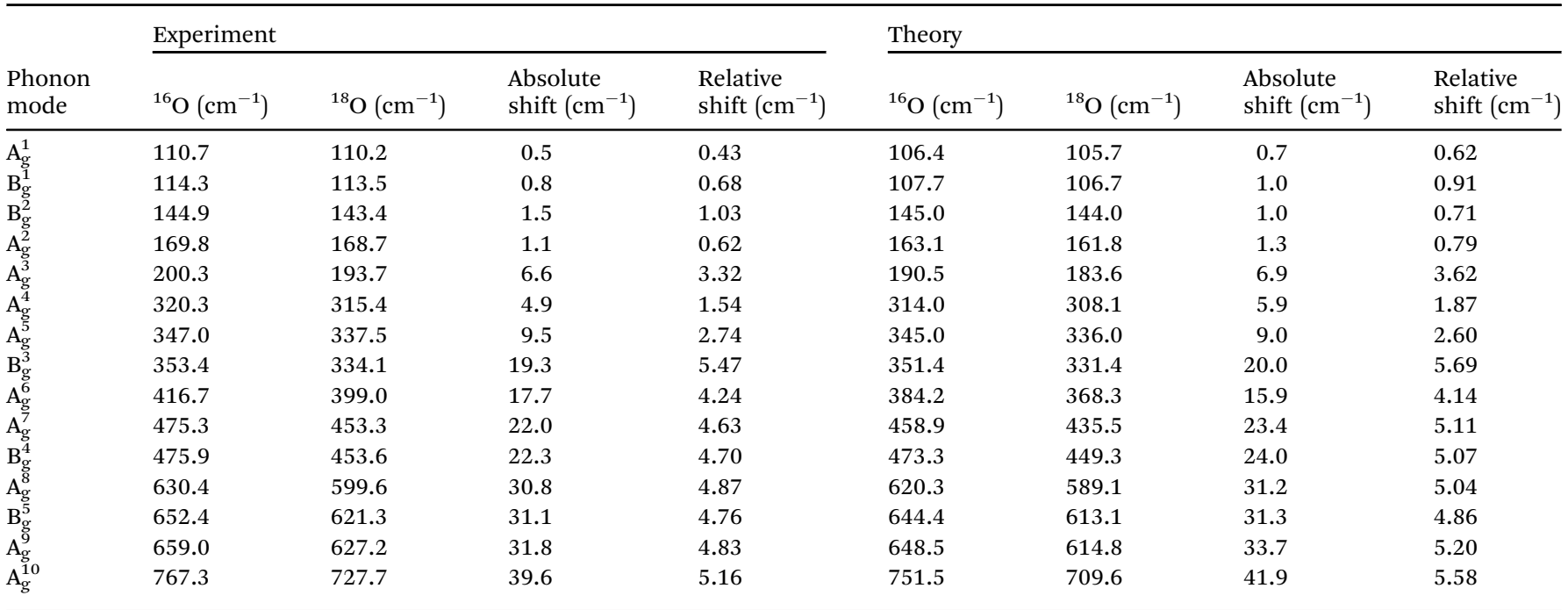

mode in the $z^{\prime}\left(x^{\prime} y^{\prime}\right) \bar{z}^{\prime}$ configuration. Using this intensity ratio, we conclude that the $\mathrm{B}_{4}$ and $\mathrm{B}_{4}^{*}$ will contribute more than $70 \%$ to the total intensity of the occurring peaks at $475.9 \mathrm{~cm}^{-1}$ and $453.6 \mathrm{~cm}^{-1}$. Hence, we assign these peaks to the $\mathrm{B}_{4}$ and $\mathrm{B}_{4}^{*}$, respectively. Analogously, the peak positions of the $A_{7}$ and $A_{7}^{*}$ are determined from the analysis of the (010) spectra in Fig. 2a with a negligible $\mathrm{B}_{4}$ or $\mathrm{B}_{4}^{*}$ intensity, respectively. Consequently, we obtain a previously unresolved mode spacing of the $\mathrm{A}_{7}$ and $\mathrm{B}_{4}$ of $0.6 \mathrm{~cm}^{-1}$ and $0.3 \mathrm{~cm}^{-1}$ for the ${ }^{16} \mathrm{O}$ and ${ }^{18} \mathrm{O}$ isotopologues.

Following this detailed analysis, we were able to determine the spectral positions of all 15 Raman-active phonon modes in the ${ }^{16} \mathrm{O}$ and ${ }^{18} \mathrm{O}$ isotopologues of $\beta-\mathrm{Ga}_{2} \mathrm{O}_{3}$ (summarized in Table 2).

While a slight change in the oxygen mass does not affect the formation of point defects during the MBE growth, altering the mass of one of the two elements of a binary oxide induces a shift in Raman modes, in which atomic vibrations of the respective element are present. Owing to the larger relative mass difference between e.g. ${ }^{16} \mathrm{O}$ and ${ }^{18} \mathrm{O}$ compared to stable $\mathrm{Ga}$ isotopes, oxygen isotopes produce an elevated frequency shift and are preferably used to study the variation of phonon frequencies in different isotopologues. ${ }^{99}$ The observed shift of Raman modes towards lower frequencies upon substitution of ${ }^{16} \mathrm{O}$ with ${ }^{18} \mathrm{O}$ corresponds to an increase in the isotopic mass. ${ }^{99,100}$

Table 2 further lists the absolute and relative frequency shift for each mode. Based on the data displayed in Table 2, Fig. 3a depicts the experimentally- (blue) and theoretically-determined (green) relative mode frequency shifts for all 15 Raman active modes. Errors in the experimental data originate from the uncertainties in the determined peak positions as described above. A qualitative agreement between experimental and theoretical data is apparent, with a slight overestimation of the frequency shifts of the majority of modes by the DFPT calculations.
The analysis of the experimental Raman mode frequency shifts in Fig. 3a reveals several noteworthy results: (i) the relative shift strongly varies between the different modes with the smallest and largest shift of $0.43 \%$ for the $\mathrm{A}_{1}$ and $5.47 \%$ for the $B_{3}$, respectively; (ii) low energy phonons between 110 and $170 \mathrm{~cm}^{-1}$ exhibit weak frequency shifts below $1.03 \%$, whereas high frequency phonons with Raman shifts above $350 \mathrm{~cm}^{-1}$ experience large relative shifts close to $5 \%$ upon $\mathrm{O}$ isotope substitution; (iii) phonons with wavenumbers between 200 and $350 \mathrm{~cm}^{-1}$ show intermediate relative shifts, which do not scale linearly with increasing phonon energy.

Fig. 4 illustrates a scheme of the Raman-active $A_{1}, A_{5}$ and $A_{10}$ modes as representatives of the low-energy phonons, phonons of intermediate energies and high-energy-phonons. Modes of A symmetry oscillate within the (010)-plane, with arrows indicating the amplitude of vibration. A scheme of all Raman-active phonon modes is presented in Fig. S3 in the ESI. $\dagger$

In order to explain the reasons for the observations (i)-(iii), we calculate the relative energy contribution of the three oxygen $\left(\mathrm{O}_{\mathrm{I}}, \mathrm{O}_{\mathrm{II}}, \mathrm{O}_{\mathrm{III}}\right)$ and two gallium $\left(\mathrm{Ga}_{\mathrm{I}}, \mathrm{Ga}_{\mathrm{II}}\right)$ lattice sites to the total phonon energy for each mode (Fig. $3 \mathrm{~b}$ ). By treating the individual atoms as harmonic oscillators, the displacement of atom $k$ in the $x$-direction is given by

$$
x_{k}(t)=A_{k} \cdot \mathrm{e}^{i \cdot \omega \cdot t},
$$

where $A_{k}$ denotes the vibration amplitude of atom $k, \omega$ is the frequency at which all atoms contributing to a specific Raman mode vibrate and $t$ is the time. The Raman frequencies $\omega$ as well as the amplitudes $A_{k}$ of the corresponding atomic vibrations are readily available from our DFPT calculations. In this approximation, each atoms kinetic energy is proportional to the product of the atoms mass and the amplitudes square of the absolute value. As a result, the energy stored in a respective Raman mode is determined by the sum of the individual kinetic energies of all 
(a)

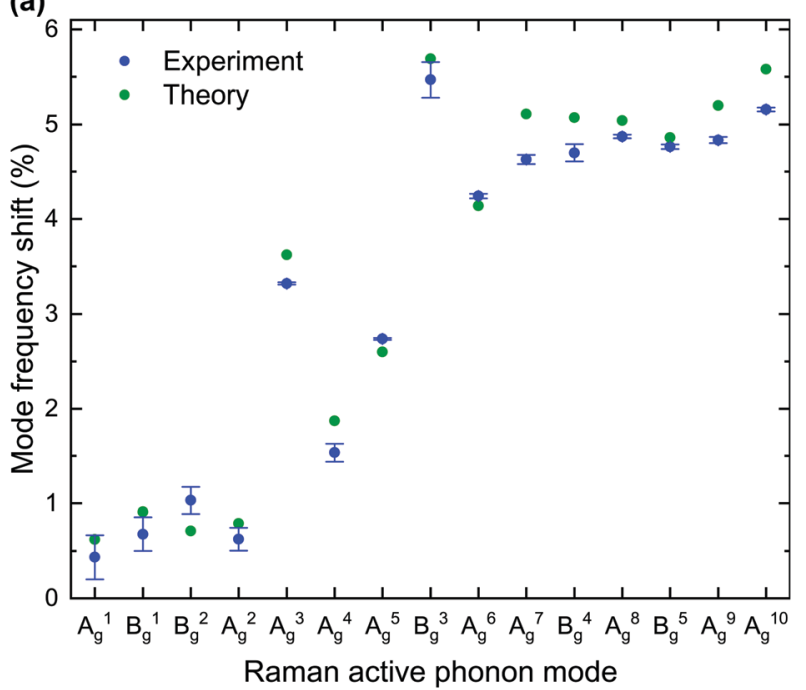

(b)

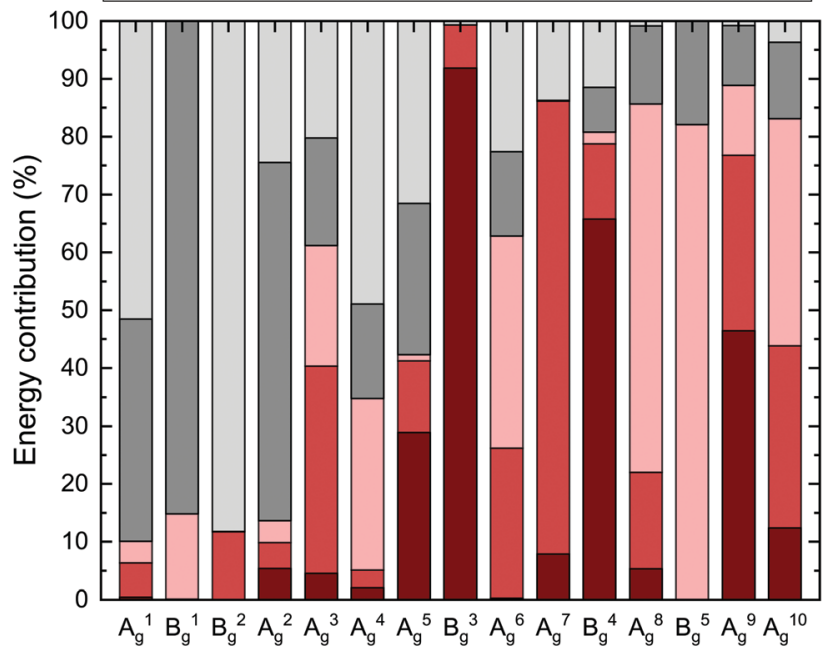

Raman active phonon mode

Fig. 3 (a) Relative mode frequency shifts of Raman modes for ${ }^{18} \mathrm{O}$ isotope substitution compared to natural isotope distribution based on experimental and theoretical data listed in Table 2. (b) Theoretical calculations of relative energy contributions to the total phonon energy corresponding to each $\mathrm{O}$ or Ga lattice site. Raman vibrations can be excited by one of the two Ga (grey) or one of the three $O$ (red) lattice sites. The height of a column indicates the relative energy contribution of the corresponding lattice site.

atoms contributing to this mode. The energy contribution of a particular $\mathrm{O}$ or Ga lattice site is given as the percentage that the sum of the corresponding lattice site atoms contributes to the total kinetic energy of this mode.

The size of the relative frequency shift is dependent upon the contribution of $\mathrm{O}$ atoms to the mode intensity. While a large relative frequency shift implies the dominance of $\mathrm{O}$ lattice site oscillations within a vibrational mode, a small shift corresponds to the absence of the same. The occurrence of a frequency shift in each of the 15 Raman modes suggests that $\mathrm{O}$ vibrations contribute to each phonon mode, which is confirmed by the fact that $\mathrm{O}$ lattice sites are present in each mode's energy contribution. Hence, pure Ga-Ga vibrations do not occur.
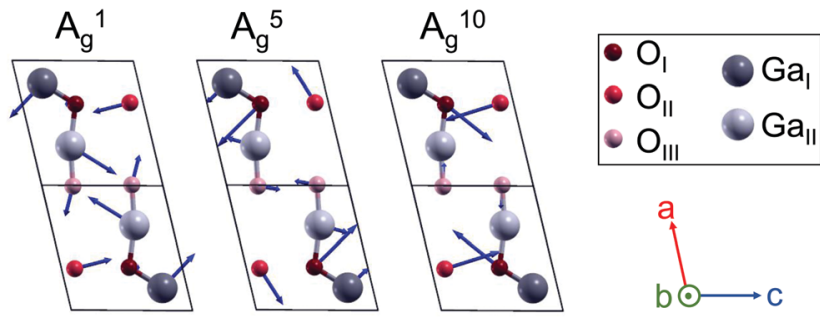

Fig. 4 Scheme of the Raman-active $A_{1}, A_{5}$ and $A_{10}$ modes within the primitive unit cell of $\beta-\mathrm{Ga}_{2} \mathrm{O}_{3}$. Modes are shown in projection on the (010)/ $b$-plane. Arrows indicate the displacements of basis atoms, with lengths denoting the amplitude of vibration. Different red and grey colours indicate individual $\mathrm{O}$ and $\mathrm{Ga}$ lattice site atoms.

The smallest relative frequency shifts seen in the lowfrequency $\mathrm{A}_{1}, \mathrm{~B}_{1}, \mathrm{~B}_{2}$ and $\mathrm{A}_{2}$ modes correspond to a comparatively low energy contribution from $\mathrm{O}$ lattice sites to the respective modes. An increased frequency shift relative to the low-frequency modes is distinctive of the $A_{3}, A_{4}, A_{5}$ and $A_{6}$ modes. O lattice sites are expected to contribute $61 \%$ to the energy of the $A_{3}$, which is reflected in an observed relative frequency shift of $3.32 \%$. Conversely, a lower frequency shift of $1.54 \%$ or $2.74 \%$ for the $\mathrm{A}_{4}$ or $\mathrm{A}_{5}$ stems from a less prominent energy contribution from $\mathrm{O}$ lattice sites. Oxygen lattice site vibrations are predominant in the $\mathrm{B}_{3}$ and the remaining highfrequency modes from $\mathrm{A}_{7}$ onward and vary between $81 \%$ or $99 \%$ for the $B_{4}$ or $B_{3}$, respectively. Correspondingly, these modes exhibit the greatest mode frequency shifts, with the largest shift observed for the $\mathrm{B}_{3}$.

Finally, we address the impact of oxygen isotope substitutions on the three distinct oxygen lattice sites. A direct comparison of the observed mode frequency shifts and phonon energy contribution of individual lattice sites indicates that the size of the relative frequency shift depends on which of the three $\mathrm{O}$ lattice sites contributes to the vibrational mode. The experimental data indicate that replacing ${ }^{16} \mathrm{O}$ with ${ }^{18} \mathrm{O}$ isotopes on the $\mathrm{O}_{\mathrm{III}}$ lattice site induces an increased frequency shift compared to $\mathrm{O}_{\mathrm{I}}$ and $\mathrm{O}_{\mathrm{II}}$ :

As for the $A_{3}$ and $A_{6}$, oxygen vibrations account for $61 \%$ or $63 \%$ of the mode energy. However, the $A_{6}$, characterized by a more intense contribution from $\mathrm{O}_{\mathrm{III}}$ lattice sites and a reduced contribution from $\mathrm{O}_{\text {II }}$ sites, has an increased relative frequency shift. Equally, oxygen vibrations account for $86 \%$ in the $\mathrm{A}_{7}$ and $A_{8}$ or $89 \%$ in the $A_{9}$. Yet, the observed frequency shift is most profound for the $A_{8}$, where $\mathrm{O}_{\text {III }}$ lattice sites prevail. As for the $\mathrm{B}_{5}$, the $\mathrm{O}$ energy contribution is comprised entirely of $\mathrm{O}_{\mathrm{III}}$ sites. Despite a lower overall oxygen contribution (82\%) compared to the $A_{7}$ and $B_{4}$ modes, the $B_{5}$ is characterized by an increased relative frequency shift. While the exact values of the computed relative energy contributions may vary slightly depending on the employed XC functional, the above LDA-based conclusions also hold true for PBE, PBEsol and opt-B88.

\section{Conclusions}

In summary, we examined a homoepitaxial $\beta-\mathrm{Ga}_{2} \mathrm{O}_{3}$ thin film in the ${ }^{18} \mathrm{O}$ isotope composition, deposited on top of a substrate 
with ${ }^{16} \mathrm{O}$ isotope distribution. Polarized micro-Raman spectroscopy was carried out to record Raman spectra of both isotopologues. By probing the (010) and (201) planes, we were able to separate all 15 Raman active phonon modes and determine their phonon frequencies for both isotopologues. The replacement of ${ }^{16} \mathrm{O}$ with the heavier ${ }^{18} \mathrm{O}$ atoms resulted in a reduction of the phonon frequency. We provided the spectral positions of ${ }^{18} \mathrm{O}$ Raman modes and quantified the absolute as well as relative frequency shifts compared to ${ }^{16} \mathrm{O}$ Raman modes. Based on the comparison of experimental data with DFPT calculations of mode frequency shifts and calculation of the relative energy contributions of each lattice site to the total phonon energy, we identified and quantified the atomistic vibrations for each of the five distinct lattice sites which give rise to the 15 different Raman-active phonon modes in $\beta-\mathrm{Ga}_{2} \mathrm{O}_{3}$. A large relative frequency shift seen in the modes of higher frequencies is emblematic of modes governed predominantly by $O$ lattice site vibrations. In contrast, the low-frequency $\mathrm{A}_{1}, \mathrm{~B}_{1}, \mathrm{~B}_{2}$ and $\mathrm{A}_{2}$ modes exhibit the smallest overall shifts and hence are governed by the vibration of both $\mathrm{Ga}$ and $\mathrm{O}$ atoms. By determining dedicated Raman modes dominated by one of the three inequivalent $\mathrm{O}$-sites and relating their relative frequency shifts to the calculated relative amount each lattice site contributes to a respective mode's energy, we conclude that substituting ${ }^{16} \mathrm{O}$ with ${ }^{18} \mathrm{O}$ isotopes on the $\mathrm{O}_{\mathrm{III}}$ lattice site results in an elevated mode frequency shift compared to $\mathrm{O}_{\mathrm{I}}$ and $\mathrm{O}_{\mathrm{II}}$ lattice sites. This discovery paves the way for the identification of O-site-related defects by Raman spectroscopy in future studies. Furthermore, micro-Raman spectroscopy may be carried out to investigate the formation of oxygen vacancies in different lattice sites as a function of different synthesis (e.g. deposition, annealing) conditions.

\section{Conflicts of interest}

There are no conflicts to declare.

\section{Acknowledgements}

We acknowledge funding by the Deutsche Forschungsgemeinschaft (DFG, German Research Foundation) - project number 446185170. This work was performed in parts in the framework of GraFOx, a Leibniz-ScienceCampus partially funded by the Leibniz association. Computational resources used for the calculations were provided by the HPC of the Regional Computer Centre Erlangen (RRZE). The authors thank Maximilian Ries for fruitful discussions, Thomas Kure for experimental support, and Arne Haeger for support in graphic design.

\section{Notes and references}

1 N. Ueda, H. Hosono, R. Waseda and H. Kawazoe, Appl. Phys. Lett., 1997, 71, 933-935.
2 M. Yamaga, T. Ishikawa, M. Yoshida, T. Hasegawa, E. G. Villora and K. Shimamura, Phys. status, 2011, 8, 2621-2624.

3 S. J. Pearton, J. Yang, P. H. Cary, F. Ren, J. Kim, M. J. Tadjer and M. A. Mastro, Appl. Phys. Rev., 2018, 5, 011301.

4 S. J. Pearton, F. Ren, M. Tadjer and J. Kim, J. Appl. Phys., 2018, 124, 220901.

5 M. Higashiwaki, K. Sasaki, A. Kuramata, T. Masui and S. Yamakoshi, Phys. Status Solidi A, 2014, 211, 21-26.

6 M. Higashiwaki, K. Sasaki, A. Kuramata, T. Masui and S. Yamakoshi, Appl. Phys. Lett., 2012, 100, 013504.

7 M. Higashiwaki, A. Kuramata, H. Murakami and Y. Kumagai, J. Phys. D: Appl. Phys., 2017, 50, 333002.

8 Y. Kokubun, K. Miura, F. Endo and S. Nakagomi, Appl. Phys. Lett., 2007, 90, 031912.

9 T. Oshima, T. Okuno, N. Arai, N. Suzuki, S. Ohira and S. Fujita, Appl. Phys. Express, 2008, 1, 011202.

10 I. Cora, F. Mezzadri, F. Boschi, M. Bosi, M. Čaplovičová, G. Calestani, I. Dódony, B. Pécz and R. Fornari, CrystEngComm, 2017, 19, 1509-1516.

11 R. Roy, V. G. Hill and E. F. Osborn, J. Am. Chem. Soc., 1952, 74, 719-722.

12 S. Yoshioka, H. Hayashi, A. Kuwabara, F. Oba, K. Matsunaga and I. Tanaka, J. Phys.: Condens. Matter, 2007, 19, 346211.

13 H. von Wenckstern, Adv. Electron. Mater., 2017, 3, 1600350.

14 S. I. Stepanov, V. I. Nikolaev, V. E. Bougrov and A. E. Romanov, Rev. Adv. Mater. Sci., 2016, 44, 63-86.

15 H. He, R. Orlando, M. A. Blanco, R. Pandey, E. Amzallag, I. Baraille and M. Rérat, Phys. Rev. B: Condens. Matter Mater. Phys., 2006, 74, 195123.

16 H. He, M. A. Blanco and R. Pandey, Appl. Phys. Lett., 2006, 88, 261904.

17 P. Kroll, R. Dronskowski and M. Martin, J. Mater. Chem., 2005, 15, 3296.

18 H. Y. Playford, A. C. Hannon, E. R. Barney and R. I. Walton, Chem. - Eur. J., 2013, 19, 2803-2813.

19 J. Furthmüller and F. Bechstedt, Phys. Rev. B: Condens. Matter Mater. Phys., 2016, 93, 115204.

20 Z. Galazka, R. Uecker, K. Irmscher, M. Albrecht, D. Klimm, M. Pietsch, M. Brützam, R. Bertram, S. Ganschow and R. Fornari, Cryst. Res. Technol., 2010, 45, 1229-1236.

21 Z. Galazka, K. Irmscher, R. Uecker, R. Bertram, M. Pietsch, A. Kwasniewski, M. Naumann, T. Schulz, R. Schewski, D. Klimm and M. Bickermann, J. Cryst. Growth, 2014, 404, 184-191.

22 K. Irmscher, Z. Galazka, M. Pietsch, R. Uecker and R. Fornari, J. Appl. Phys., 2011, 110, 063720.

23 V. I. Vasyltsiv, Y. I. Rym and Y. M. Zakharko, Phys. Status Solidi, 1996, 195, 653-658.

24 E. Villora, Y. Morioka, T. Atou, T. Sugawara, M. Kikuchi and T. Fukuda, Phys. Status Solidi, 2002, 193, 187-195.

25 N. Suzuki, S. Ohira, M. Tanaka, T. Sugawara, K. Nakajima and T. Shishido, Phys. Status Solidi C, 2007, 4, 2310-2313.

26 S. Ohira, N. Suzuki, N. Arai, M. Tanaka, T. Sugawara, K. Nakajima and T. Shishido, Thin Solid Films, 2008, 516, 5763-5767. 
27 J. Zhang, B. Li, C. Xia, G. Pei, Q. Deng, Z. Yang, W. Xu, H. Shi, F. Wu, Y. Wu and J. Xu, J. Phys. Chem. Solids, 2006, 67, 2448-2451.

28 A. Kuramata, K. Koshi, S. Watanabe, Y. Yamaoka, T. Masui and S. Yamakoshi, Jpn. J. Appl. Phys., 2016, 55, $1202 \mathrm{~A} 2$.

29 V. Nikolaev, V. Maslov, S. Stepanov, A. Pechnikov, V. Krymov, I. Nikitina, L. Guzilova, V. Bougrov and A. Romanov, J. Cryst. Growth, 2017, 457, 132-136.

30 M. Mohamed, K. Irmscher, C. Janowitz, Z. Galazka, R. Manzke and R. Fornari, Appl. Phys. Lett., 2012, 101, 132106.

31 K. Suzuki, T. Okamoto and M. Takata, Ceram. Int., 2004, 30, 1679-1683.

32 S. Geller, J. Chem. Phys., 1960, 33, 676-684.

33 P. Mazzolini, A. Falkenstein, C. Wouters, R. Schewski, T. Markurt, Z. Galazka, M. Martin, M. Albrecht and O. Bierwagen, APL Mater., 2020, 8, 011107.

34 K. Sasaki, A. Kuramata, T. Masui, E. G. Víllora, K. Shimamura and S. Yamakoshi, Appl. Phys. Express, 2012, 5, 035502.

35 M. Higashiwaki, K. Konishi, K. Sasaki, K. Goto, K. Nomura, Q. T. Thieu, R. Togashi, H. Murakami, Y. Kumagai, B. Monemar, A. Koukitu, A. Kuramata and S. Yamakoshi, Appl. Phys. Lett., 2016, 108, 133503.

36 S. Rafique, M. R. Karim, J. M. Johnson, J. Hwang and H. Zhao, Appl. Phys. Lett., 2018, 112, 052104.

37 M.-Y. Tsai, O. Bierwagen, M. E. White and J. S. Speck, J. Vac. Sci. Technol., A, 2010, 28, 354-359.

38 G. Wagner, M. Baldini, D. Gogova, M. Schmidbauer, R. Schewski, M. Albrecht, Z. Galazka, D. Klimm and R. Fornari, Phys. Status Solidi A, 2014, 211, 27-33.

39 H. Murakami, K. Nomura, K. Goto, K. Sasaki, K. Kawara, Q. Tu Thieu, R. Togashi, Y. Kumagai, M. Higashiwaki, A. Kuramata, S. Yamakoshi, B. Monemar and A. Koukitu, Appl. Phys. Express, 2015, 8, 015503.

40 S.-D. Lee, K. Kaneko and S. Fujita, Jpn. J. Appl. Phys., 2016, 55, $1202 B 8$.

41 R. Schewski, K. Lion, A. Fiedler, C. Wouters, A. Popp, S. V. Levchenko, T. Schulz, M. Schmidbauer, S. Bin Anooz, R. Grüneberg, Z. Galazka, G. Wagner, K. Irmscher, M. Scheffler, C. Draxl and M. Albrecht, APL Mater., 2019, 7, 022515.

42 E. G. Víllora, K. Shimamura, Y. Yoshikawa, T. Ujiie and K. Aoki, Appl. Phys. Lett., 2008, 92, 202120.

43 M. Orita, H. Ohta, M. Hirano and H. Hosono, Appl. Phys. Lett., 2000, 77, 4166-4168.

44 E. Ahmadi, O. S. Koksaldi, S. W. Kaun, Y. Oshima, D. B. Short, U. K. Mishra and J. S. Speck, Appl. Phys. Express, 2017, 10, 041102.

45 W. Zhou, C. Xia, Q. Sai and H. Zhang, Appl. Phys. Lett., 2017, 111, 242103.

46 S. C. Siah, R. E. Brandt, K. Lim, L. T. Schelhas, R. Jaramillo, M. D. Heinemann, D. Chua, J. Wright, J. D. Perkins, C. U. Segre, R. G. Gordon, M. F. Toney and T. Buonassisi, Appl. Phys. Lett., 2015, 107, 252103.

47 T. Onuma, S. Fujioka, T. Yamaguchi, M. Higashiwaki, K. Sasaki, T. Masui and T. Honda, Appl. Phys. Lett., 2013, 103, 041910.
48 M. Baldini, M. Albrecht, A. Fiedler, K. Irmscher, R. Schewski and G. Wagner, ECS J. Solid State Sci. Technol., 2017, 6, Q3040-Q3044.

49 J. B. Varley, J. R. Weber, A. Janotti and C. G. Van de Walle, Appl. Phys. Lett., 2010, 97, 142106.

50 S. Lany, APL Mater., 2018, 6, 046103.

51 J. L. Lyons, Semicond. Sci. Technol., 2018, 33, 05LT02.

52 J. Lee, S. Ganguli, A. K. Roy and S. C. Badescu, J. Chem. Phys., 2019, 150, 174706.

53 T. Zacherle, P. C. Schmidt and M. Martin, Phys. Rev. B: Condens. Matter Mater. Phys., 2013, 87, 235206.

54 A. Kyrtsos, M. Matsubara and E. Bellotti, Phys. Rev. B, 2017, 95, 245202.

55 P. Deák, Q. Duy Ho, F. Seemann, B. Aradi, M. Lorke and T. Frauenheim, Phys. Rev. B, 2017, 95, 075208.

56 H. Peelaers, J. L. Lyons, J. B. Varley and C. G. Van de Walle, APL Mater., 2019, 7, 022519.

57 K. Momma and F. Izumi, J. Appl. Crystallogr., 2011, 44, 1272-1276.

58 M. R. Wagner, T. P. Bartel, R. Kirste, A. Hoffmann, J. Sann, S. Lautenschläger, B. K. Meyer and C. Kisielowski, Phys. Rev. B: Condens. Matter Mater. Phys., 2009, 79, 035307.

59 A. Kaschner, U. Haboeck, M. Strassburg, M. Strassburg, G. Kaczmarczyk, A. Hoffmann, C. Thomsen, A. Zeuner, H. R. Alves, D. M. Hofmann and B. K. Meyer, Appl. Phys. Lett., 2002, 80, 1909-1911.

60 C. Kranert, C. Sturm, R. Schmidt-Grund and M. Grundmann, Sci. Rep., 2016, 6, 35964.

61 J. S. Reparaz, E. Chavez-Angel, M. R. Wagner, B. Graczykowski, J. Gomis-Bresco, F. Alzina and C. M. Sotomayor Torres, Rev. Sci. Instrum., 2014, 85, 034901.

62 E. Chávez-Ángel, J. S. Reparaz, J. Gomis-Bresco, M. R. Wagner, J. Cuffe, B. Graczykowski, A. Shchepetov, H. Jiang, M. Prunnila, J. Ahopelto, F. Alzina and C. M. Sotomayor Torres, APL Mater., 2014, 2, 012113.

63 D. Dohy, G. Lucazeau and A. Revcolevschi, J. Solid State Chem., 1982, 45, 180-192.

64 D. Machon, P. F. McMillan, B. Xu and J. Dong, Phys. Rev. B: Condens. Matter Mater. Phys., 2006, 73, 094125.

65 B. Liu, M. Gu and X. Liu, Appl. Phys. Lett., 2007, 91, 172102. 66 T. Onuma, S. Fujioka, T. Yamaguchi, Y. Itoh, M. Higashiwaki, K. Sasaki, T. Masui and T. Honda, J. Cryst. Growth, 2014, 401, 330-333.

67 M. Schubert, R. Korlacki, S. Knight, T. Hofmann, S. Schöche, V. Darakchieva, E. Janzén, B. Monemar, D. Gogova, Q.-T. Thieu, R. Togashi, H. Murakami, Y. Kumagai, K. Goto, A. Kuramata, S. Yamakoshi and M. Higashiwaki, Phys. Rev. B, 2016, 93, 125209.

68 C. Kranert, C. Sturm, R. Schmidt-Grund and M. Grundmann, Phys. Rev. Lett., 2016, 116, 127401.

69 K. A. Mengle and E. Kioupakis, AIP Adv., 2019, 9, 015313.

70 L. Kavan, M. Zukalova, M. Ferus, J. Kürti, J. Koltai and S. Civiš, Phys. Chem. Chem. Phys., 2011, 13, 11583.

71 O. Frank, M. Zukalova, B. Laskova, J. Kürti, J. Koltai and L. Kavan, Phys. Chem. Chem. Phys., 2012, 14, 14567. 
72 H. Kaftelen, K. Ocakoglu, R. Thomann, S. Tu, S. Weber and E. Erdem, Phys. Rev. B: Condens. Matter Mater. Phys., 2012, 86, 014113.

73 E. Erdem, J. Alloys Compd., 2014, 605, 34-44.

74 Ü. Özgür, Y. I. Alivov, C. Liu, A. Teke, M. A. Reshchikov, S. Doğan, V. Avrutin, S. J. Cho and H. Morkoç, J. Appl. Phys., 2005, 98, 041301.

75 M. D. McCluskey and S. J. Jokela, J. Appl. Phys., 2009, 106, 71101.

76 Y. M. Strzhemechny, H. L. Mosbacker, S. H. Goss, D. C. Look, D. C. Reynolds, C. W. Litton, N. Y. Garces, N. C. Giles, L. E. Halliburton, S. Niki and L. J. Brillson, J. Electron. Mater., 2005, 34, 399-403.

77 C. G. Van De Walle, Phys. B, 2001, 308-310, 899-903.

78 E. A. Davis, S. P. Cottrell, P. J. King, J. S. Lord, J. M. Gil, H. V. Alberto, R. C. Vilão, J. Piroto Duarte, N. Ayres de Campos, A. Weidinger, R. L. Lichti, S. J. Irvine and S. F. Cox, Phys. Rev. Lett., 2001, 86, 2601-2604.

79 A. Kohan, G. Ceder, D. Morgan and C. G. Van de Walle, Phys. Rev. B: Condens. Matter Mater. Phys., 2000, 61, 15019-15027.

80 B. K. Meyer, J. Sann, S. Lautenschläger, M. R. Wagner and A. Hoffmann, Phys. Rev. B: Condens. Matter Mater. Phys., 2007, 76, 184120.

81 A. Kaschner, U. Haboeck, M. Strassburg, M. Strassburg, G. Kaczmarczyk, A. Hoffmann, C. Thomsen, A. Zeuner, H. R. Alves, D. M. Hofmann and B. K. Meyer, Appl. Phys. Lett., 2002, 80, 1909-1911.

82 F. Friedrich and N. H. Nickel, Appl. Phys. Lett., 2007, 91, 111903.

83 F. Friedrich, M. A. Gluba and N. H. Nickel, Appl. Phys. Lett., 2009, 95, 141903.

84 M. A. Gluba, N. H. Nickel and N. Karpensky, Phys. Rev. B: Condens. Matter Mater. Phys., 2013, 88, 245201.

85 L. Artús, R. Cuscó, E. Alarcón-Lladó, G. González-Díaz, I. Mártil, J. Jiménez, B. Wang and M. Callahan, Appl. Phys. Lett., 2007, 90, 181911.

86 J. B. Wang, H. M. Zhong, Z. F. Li and W. Lu, Appl. Phys. Lett., 2006, 88, 101913.
87 C. Bundesmann, N. Ashkenov, M. Schubert, D. Spemann, T. Butz, E. M. Kaidashev, M. Lorenz and M. Grundmann, Appl. Phys. Lett., 2003, 83, 1974-1976.

88 P. Mazzolini, V. Russo, C. S. Casari, T. Hitosugi, S. Nakao, T. Hasegawa and A. Li Bassi, J. Phys. Chem. C, 2016, 120, 18878-18886.

89 P. Vogt, O. Brandt, H. Riechert, J. Lähnemann and O. Bierwagen, Phys. Rev. Lett., 2017, 119, 196001.

90 O. Bierwagen, P. Vogt and P. Mazzolini, in Gallium OxideMaterials Properties, Crystal Growth, and Devices, ed. M. Higashiwaki and S. Fujita, Springer International Publishing, Cham, 2020, vol. 293, ch. 6, pp. 95-121.

91 P. Mazzolini, P. Vogt, R. Schewski, C. Wouters, M. Albrecht and O. Bierwagen, APL Mater., 2019, 7, 022511.

92 P. Giannozzi, S. Baroni, N. Bonini, M. Calandra, R. Car, C. Cavazzoni, D. Ceresoli, G. L. Chiarotti, M. Cococcioni, I. Dabo, A. D. Corso, S. de Gironcoli, S. Fabris, G. Fratesi, R. Gebauer, U. Gerstmann, C. Gougoussis, A. Kokalj, M. Lazzeri, L. Martin-Samos, N. Marzari, F. Mauri, R. Mazzarello, S. Paolini, A. Pasquarello, L. Paulatto, C. Sbraccia, S. Scandolo, G. Sclauzero, A. P. Seitsonen, A. Smogunov, P. Umari and R. M. Wentzcovitch, J. Phys.: Condens. Matter, 2009, 21, 395502.

93 D. R. Hamann, Phys. Rev. B, 2013, 88, 085117.

94 M. J. van Setten, M. Giantomassi, E. Bousquet, M. J. Verstraete, D. R. Hamann, X. Gonze and G.M. Rignanese, Comput. Phys. Comm., 2018, 226, 39-54.

95 L. He, F. Liu, G. Hautier, M. J. T. Oliveira, M. A. L. Marques, F. D. Vila, J. J. Rehr, G.-M. Rignanese and A. Zhou, Phys. Rev. B: Condens. Matter Mater. Phys., 2014, 89, 064305.

96 J. Åhman, G. Svensson and J. Albertsson, Acta Crystallogr., Sect. C: Cryst. Struct. Commun., 1996, 52, 1336-1338.

97 K. Yamaguchi, Solid State Commun., 2004, 131, 739-744.

98 P. Mazzolini and O. Bierwagen, J. Phys. D: Appl. Phys., 2020, 53, 354003.

99 M. Cardona and M. L. W. Thewalt, Rev. Mod. Phys., 2005, 77, 1173-1224.

100 M. D. McCluskey, J. Appl. Phys., 2000, 87, 3593-3617. 